\title{
Research of Quartz Tuning Fork Tip Length Influence on the Shear Force Imaging in Liquids
}

\author{
V. LENDRAITIS \\ Kaunas University of Technology, Studentu 65, 51369 Kaunas, Lithuania, E-mail: vitas_l@hotmail.com \\ cross $^{\text {ref }}$ http://dx.doi.org/10.5755/j01.mech.24.1.19059
}

\section{Introduction}

Shear force detection method has been widely used for regulating the tip-sample distance in scanning near-field optical microscopy (SNOM) since its implementation. In this technique, an optical fibre tip is fixed to a vibrating element, which is excited near its mechanical resonance frequency in such a way that the tip vibrates parallel (shear force mode) or perpendicular tapping mode) to the sample surface. As the tip approaches close to the sample surface, the tip-sample interaction forces experienced by the sensor are detected as a shift in resonance frequency and changes in vibrational amplitude and phase. These signals are used to maintain the tip-sample distance of the SNOM during scanning. The imaging performance of the dynamic distance control is mainly determined by the response sensitivity of the sensor to the tip-sample interaction forces. It has been shown that the minimum detectable force has an inverse dependence on the square root of the sensor's quality factor $\mathrm{Q}$ and dithering frequency $[1,2]$. Sometimes the sensor has to be immersed in an aqueous medium. In these cases, the force detection sensitivity is considerably degraded owing to capillary and viscous damping. Therefore, imaging of such samples becomes difficult, especially in the shear force detection mode.

In this work, we investigate the possibility to use a tuning-fork with a long $(5 \mathrm{~mm})$ tungsten tip to operate in liquid environments. We have investigated the damping of the tip oscillation as a function of tip length in an air and in a liquid at higher modes of quartz tuning fork (QTF).

The tuning-fork and a tip oscillation amplitude-frequency dependence in air and a tip in contact with surface were investigated using Finite Element Modeling and experimentally.

\section{Theoretical aspects of electrochemical etching tungs- ten tips}

Under the influence of a high electrostatic field, electrons can be emitted from the surface of a tungsten tip into electrolyte. This purely quantum mechanical phenomenon is called field emission, and it proved to be a proper tool for the characterization of our tips since it allowed us to gain some useful insights into the sharpness of tungsten tips of our probe [3].

The electrostatic field at the tip surface increases at the regions of high curvature. If voltage $V$ is applied to asphere of radius $R$, the electrostatic field $\mathrm{F}$ at its surface is given by:

$$
F=V / R \text {. }
$$

The tip is composed of a sphere attached to a conical shank. The electrostatic field that results at the apex surface will be lower than predicted by Eq. (1) because the presence of the shank yields a modification in the field lines distribution. The surface electrostatic field for a tip-shaped object can then be approximated by:

$$
F=V / k R \text {, }
$$

where: $k$ is the field reduction factor and $R$ is the tip radius. From Eq. (2), it can be seen that for a given applied voltage and field reduction factor, a smaller tip apex radius will yield a higher electrostatic field. From the Folver Nordheim equations [4] we also know that a higher electrostatic field will produce a higher current density of field emission $\left(\mathrm{A} / \mathrm{mm}^{2}\right)$ :

$$
i=6.2 \cdot 10^{-6} \frac{\mu / \varphi^{1 / 2}}{\mu+\varphi} F^{2} \times \exp \left[6.8 \cdot 10^{7} \frac{\varphi^{3 / 2}}{F}\right],
$$

for $\mu(\mathrm{eV})$ and $\varphi(\mathrm{eV})$ and $F(\mathrm{~V} / \mathrm{mm})$. Here $\mu$ is a Fermi level and $\varphi$ is a metal's work function. Then:

$i=6.2 \cdot 10^{-6} \frac{\mu / \varphi^{1 / 2}}{\alpha^{2}(\mu+\varphi)} F^{2} \times \exp \left[6.8 \cdot 10^{7} \frac{\varphi^{3 / 2} \alpha}{F}\right]$

where: $\alpha$ has a value comprised of varying 0 and 1 . If Eq. (4) multiplied by the total field emitting area $\alpha\left(\mathrm{mm}^{2}\right)$ and Eq. (2) is used to express the electrostatic field $F$, the total field emitted current $I(\mathrm{~A})$ is obtained as a function of the tip radius $R$ ( $\mathrm{mm})$ :

$$
\begin{aligned}
& i=\alpha 6.2 \cdot 10^{-6} \frac{\mu / \varphi^{1 / 2}}{\alpha^{2}(\mu+\varphi)} \frac{V^{2}}{(k R)^{2}} \times \\
& \times \exp \left[-6.8 \cdot 10^{7} \frac{\varphi^{3 / 2} \alpha k R}{V}\right] .
\end{aligned}
$$

Eq. (5) provides us with an explicit description of the relationship between tip sharpness and field emission data: for any tip voltage, the smaller the tip apex radius, the higher the total field emitted current. This principle is the essential factor behind our quick tip sharpness test.

The tip radius can be estimated by a direct application of the Fowler-Northeim theory on field emission.

If Eq. (5) is divided by $V^{2}$ and the natural logarithm on both sides, are obtained 


$$
\begin{aligned}
& \ln \frac{I}{V^{2}}=\ln \left[\alpha 6.2 \cdot 10^{6} \frac{(\mu / \varphi)^{1 / 2}}{\alpha^{2}(\mu+\varphi)(k R)^{2}}\right]- \\
& -6.8 \cdot 10^{7} \frac{\varphi^{3 / 2} \alpha k R}{V} .
\end{aligned}
$$

Eq. (6) can yield a straight line of $\ln I / V^{2}$ versus $1 / V$ that will yield a straight line of slope $-6.8 \cdot 10^{7} \varphi^{3 / 2} \alpha k R$. This particular way of graphing field emission data is called a Fowler - Northeim plot and simply requires to record the total field emission current for various applied voltages. Provided that the values of $\varphi, \alpha$ and $k$ are known, it is then a straightforward matter to evaluate the tip radius from the slope of a Fowler-Northeim plot [4].

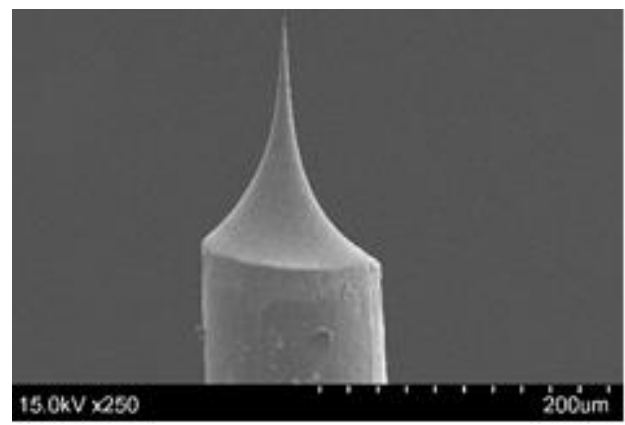

Fig. 1 Optical microscope image of the sharpened probe tip end

Monitoring the etching current drawn from tungsten tips as a function of applied voltage is a convenient way to characterize the sharpness of tips of our probe.

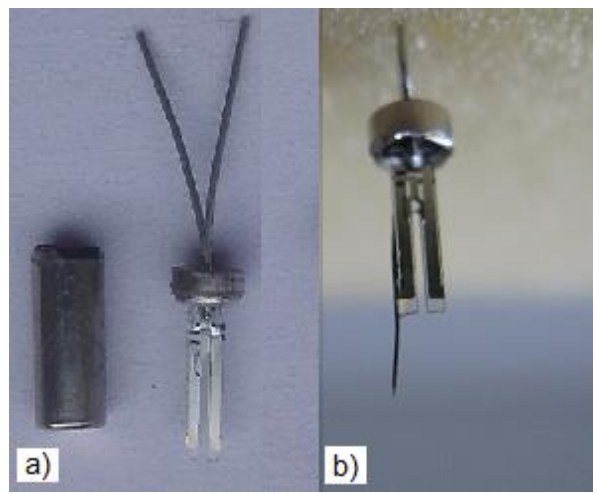

Fig. 2 Photograph of the QTF probe: $a$ - general view; $b$ sensor used for shear force microscope probe when is parallel to quartz fork

The experimental setup permitting the acquisition of such field emission data is quite simple.

The use of electrochemical etching has enabled us to achieve the sharpness of the tungsten tip where the sphere diameter is smaller than $50 \mu \mathrm{m}$ (Fig. 1). The force sensor for shear force microscope (SFM) has been produced by gluing a sharpened probe to a QTF or a resonator of some other type [2].

The gluing quality is ensured by the usage of a specially constructed $\mathrm{x}, \mathrm{y}, \mathrm{z}$ coordinate manipulator placed in the observation zone of microscope. The QTF and sensor are shown in Fig. 2.

\section{Modelling the quartz tuning fork}

The probe must be within a few nanometers of the surface because of the interaction volume of the highly localized field enhancement. In order to guarantee this tipsample interaction distance, an SFM is used. This is clearly seen in Fig. 3. SFM have the capacity to hold a probe just several nanometers above the surface of the sample. The amplitude and phase of a quartz tuning fork are monitored by high - speed electronics, while the Z-piezo is controlled by a Proportional - Integral - Differential (PID) feedback loop. The function of the feedback loop is to maintain a constant height of the imaging probe above the sample topography while scanning takes place.

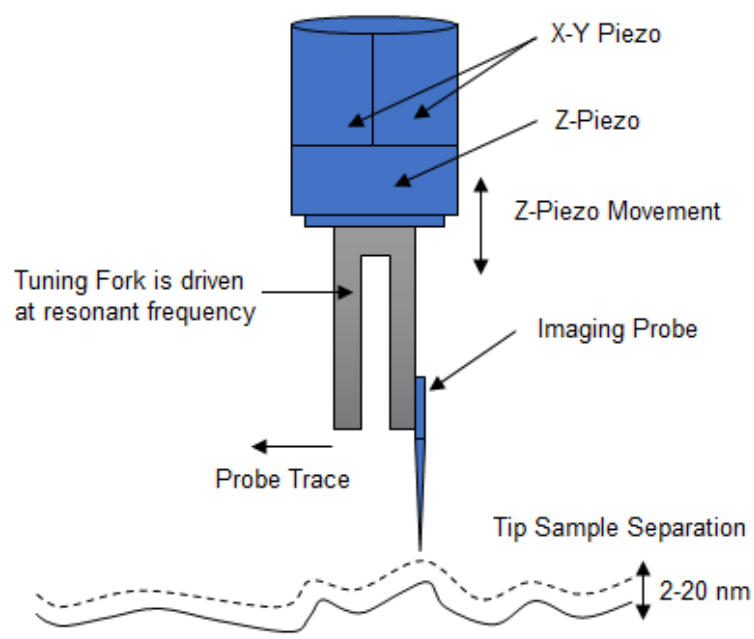

Fig. 3 The drawing of the tuning fork SFM

The use of the imaging probes that are mounted onto quartz tuning forks make angstrom level resolutions possible [5]. The sample is placed under the probe to ensure maintenance of laser illumination and probe alignment during the raster scanning of the sample.

In this work, a finite element model (FEM) of the QTF is suggested. In order to make a precise 3D model, the dimensions of the QTF are set in accordance with the measurements carried out in an optical microscope. In contrast to commercial AFM sensors where cantilevers present a rotation in relation to the $\mathrm{X}$ coordinate, the tuning fork model rotates in relation to the $\mathrm{Z}$ coordinate; thus, the width $(\mathrm{T})$ and the thickness (W) are defined in opposite ways compared to AFM cantilevers (Fig. 4.)

As it has been mention previously, the QTF is based on piezoelectric phenomena. Therefore, linear piezoelectricity equations of elasticity have to be defined and coupled to the electrostatic charge by means of piezoelectric constants [6]:

$$
\begin{aligned}
& {\left[\begin{array}{c}
\varepsilon_{p} \\
D_{i}
\end{array}\right]=\left[\begin{array}{cc}
S_{p q}^{E} & d_{p k} \\
d_{i q} & \varepsilon_{i k}^{\sigma}
\end{array}\right]\left[\begin{array}{c}
\sigma_{q} \\
E_{k}
\end{array}\right],} \\
& {\left[\begin{array}{c}
\sigma_{p} \\
D_{i}
\end{array}\right]=\left[\begin{array}{cc}
Y_{p q}^{E} & e_{p q} \\
e_{i q} & \varepsilon_{i k}^{\varepsilon}
\end{array}\right]\left[\begin{array}{c}
\varepsilon_{q} \\
E_{k}
\end{array}\right],}
\end{aligned}
$$


where: $\left\{D_{i}\right\}$ is electric flux density vector, $\left\{\sigma_{p}\right\}$ is stress vector, $\left\{E_{k}\right\}$ is elastic strain vector, $\left\{Y_{p q}^{E}\right\}$ is elasticity matrix, $\left\{e_{p k}\right\}$ is piezoelectric stress matrix, $\left\{\varepsilon_{q}\right\}$ is electric field intensity vector, $\left\{\varepsilon_{i k}^{\varepsilon}\right\}$ is dielectric matrix.

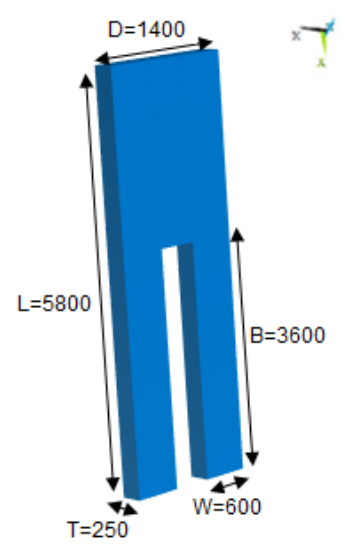

Fig. 4 Dimensions and geometry of QTF $(\mu \mathrm{m})$

The different physical properties of quartz have been extensively studied [7, 8]. However, there are insignificant differences in elastic, piezoelectric, and dielectric permittivity matrices among various reported study results. In the present work, properties from [8] have been selected because more complex measurement techniques based on resonance ultrasound spectroscopy are used to determine the values.

The piezoelectric constant matrix, which allows the structural and electrical behaviour of the material to be coupled, is defined as follows:

$$
\left[e_{p k}\right]=\left[\begin{array}{ccc}
-0.151 & 0 & 0 \\
0.151 & 0 & 0 \\
0 & 0 & 0 \\
0.061 & 0 & 0 \\
0 & -0.061 & 0 \\
0 & 0.151 & 0
\end{array}\right] \frac{c}{m^{2}} .
$$
follows:

The piezoelectric coefficients can be described as

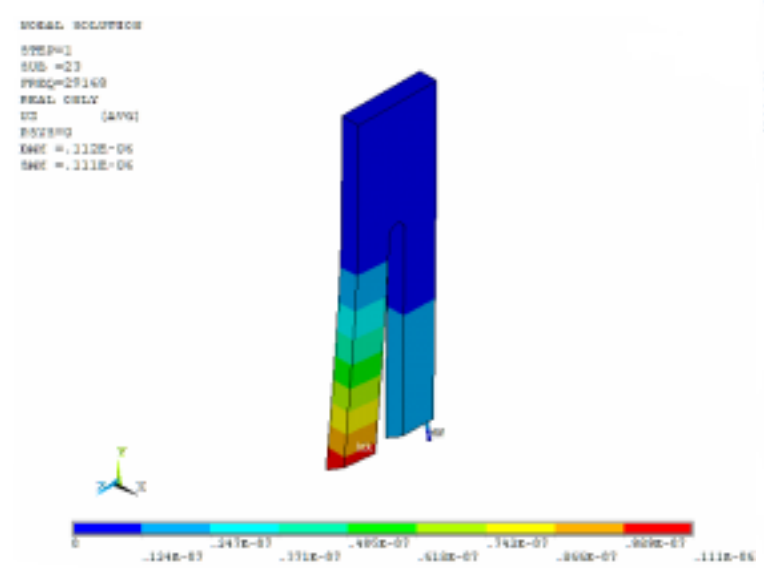

$$
\begin{gathered}
{\left[Y_{p q}^{E}\right]=\left[\begin{array}{ccc}
86.76 & 6.86 & 11.85 \\
6.86 & 86.76 & 11.85 \\
11.85 & 11.85 & 105.46 \\
-18.02 & 18.02 & 0 \\
0 & 0 & 0 \\
0 & 0 & 0 \\
-18.02 & 0 & 0 \\
-18.02 & 0 & 0 \\
0 & 0 & 0 \\
58.14 & 0 & 0 \\
0 & 58.14 & -18.02 \\
0 & 18.02 & 39.95
\end{array}\right] \mathrm{GPa} .}
\end{gathered}
$$

The piezoelectric behaviour of the material is achieved by using the element type SOLID226 in ANSYS. The model is defined in MKS units in line with ANSYS nomenclature.

\section{Results and discussion}

Below is a general view of the QTF (Fig. 5). The difference in tuning fork oscillation resonance frequency with the free tip in air and with the tip in contact with surface was used as a measure to evaluate the tip length influence on shear force sensor sensivity to acting forces (Fig. 6 - 9). The tungsten tip diameter $120 \mu \mathrm{m}$ was used for simulations.

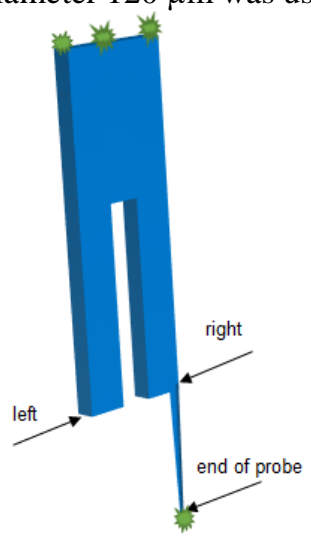

Fig. 5 General view of the QTF

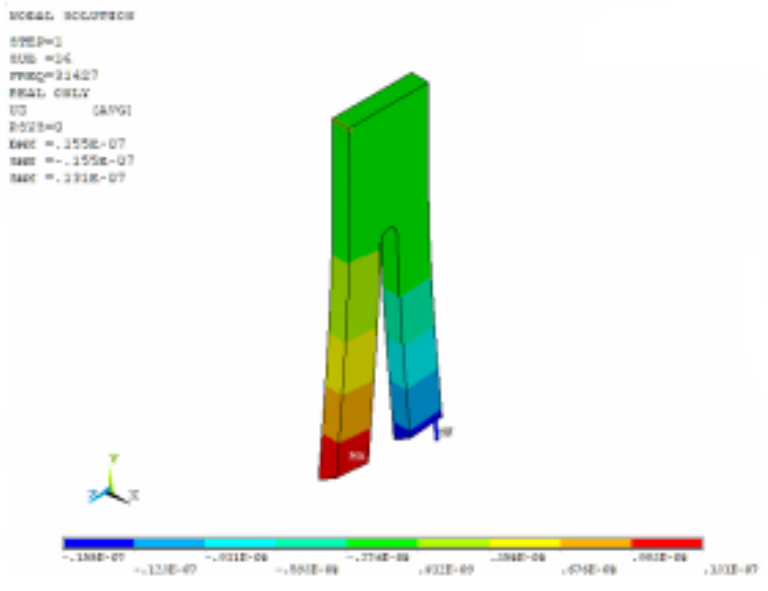

b

Fig. 6 Continued on next page 


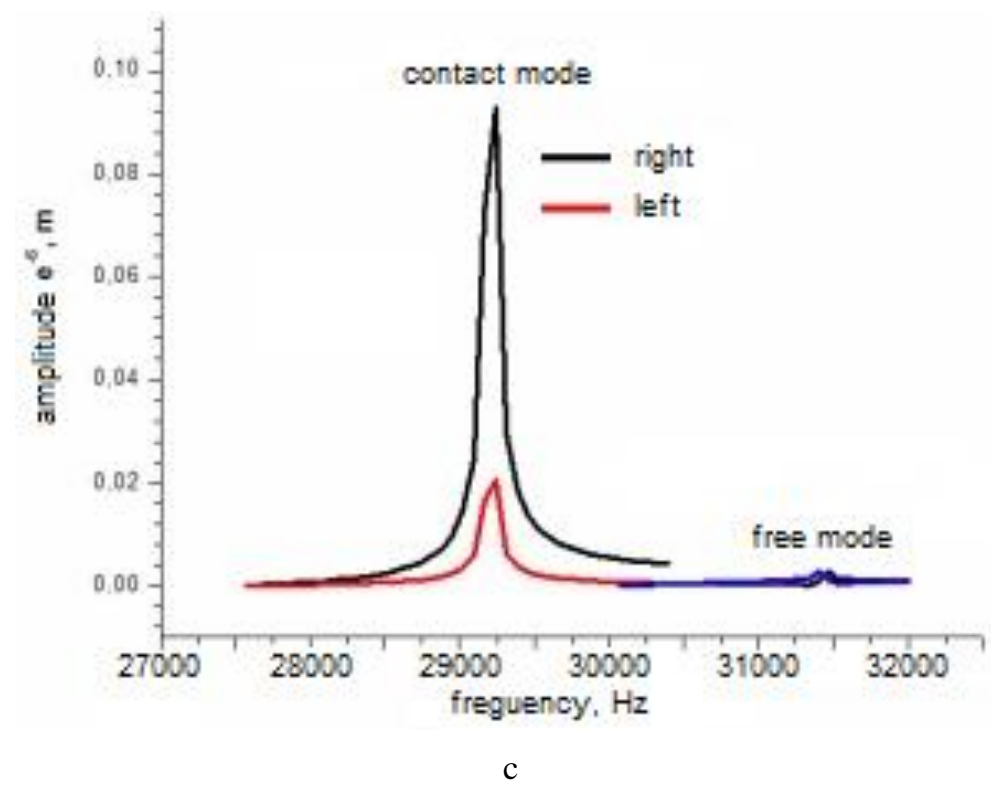

Fig. 6 Tuning fork oscillation resonance frequency $32 \mathrm{kHz}$, tip length $0.4 \mathrm{~mm}$ a) contact with surface b) free contact c) amplitude-frequency characteristic

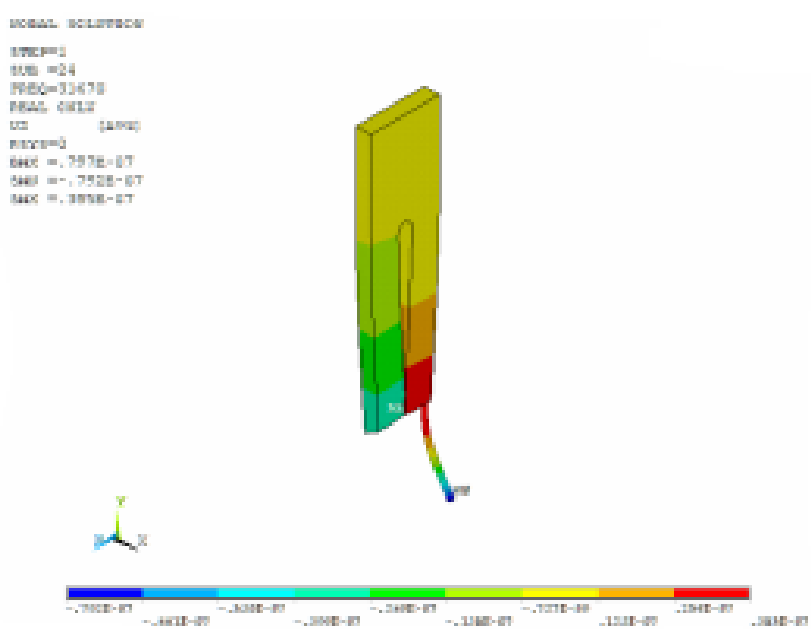

a

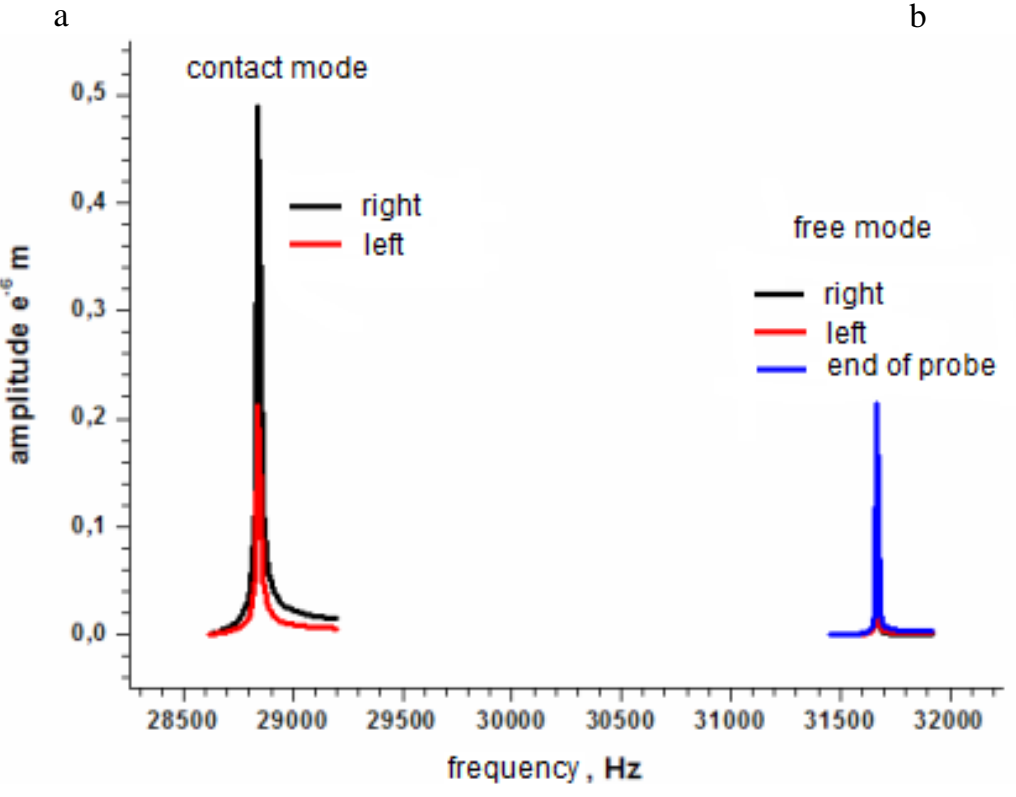

$\mathrm{C}$

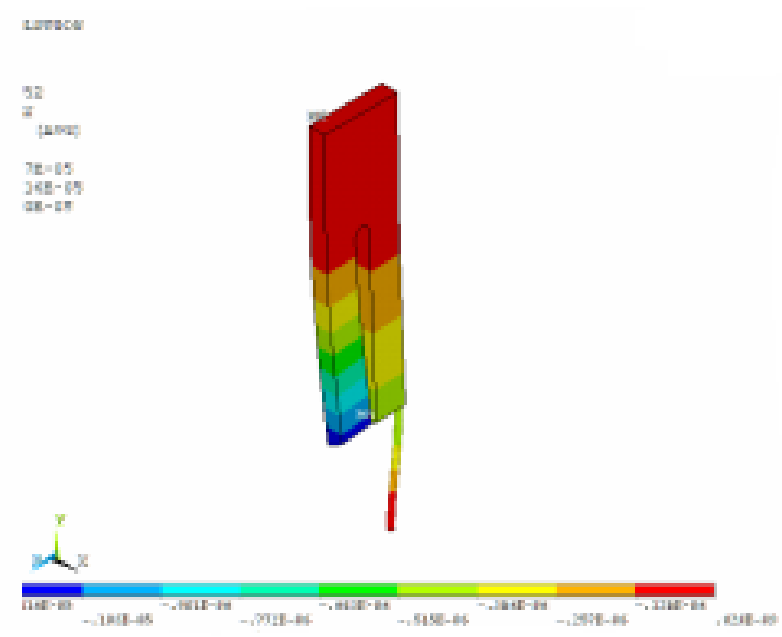

$\mathrm{b}$

Fig. 7 Tuning fork oscillation resonance frequency $32 \mathrm{kHz}$, tip length $2 \mathrm{~mm}$ a) contact with surface b) free contact c) amplitude-frequency characteristic 


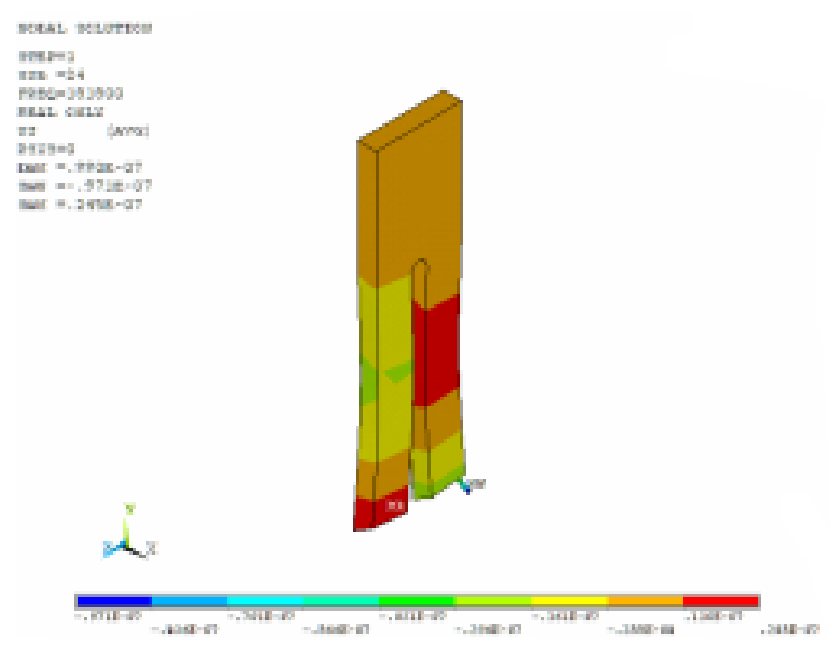

a

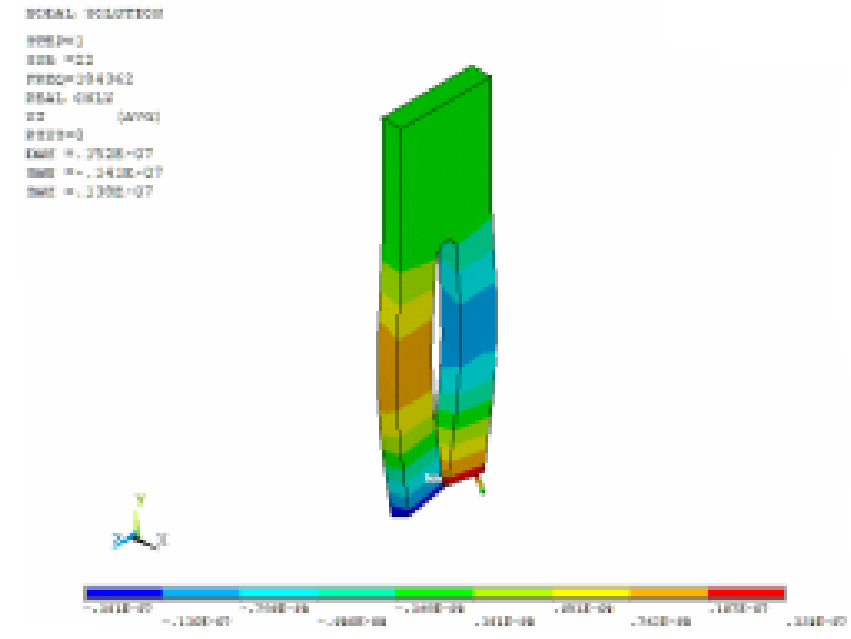

b

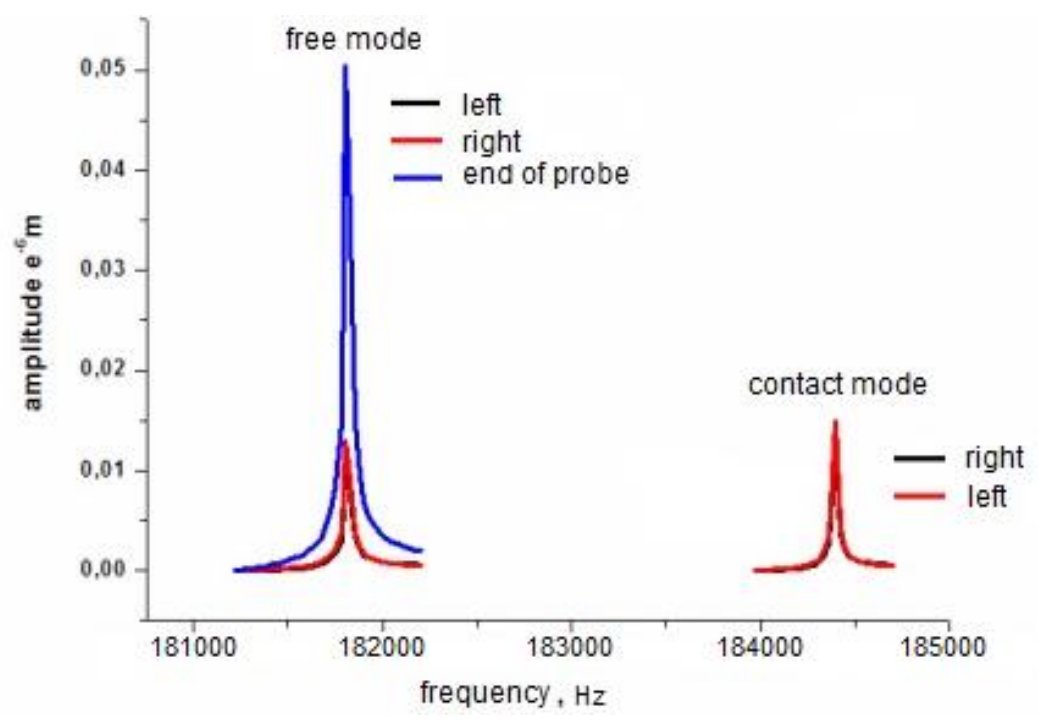

$\mathrm{c}$

Fig. 8 Tuning fork oscillation resonance frequency $190 \mathrm{kHz}$, tip length $0.4 \mathrm{~mm}$ a) free contact b) contact with surface c) amplitude-frequency characteristic

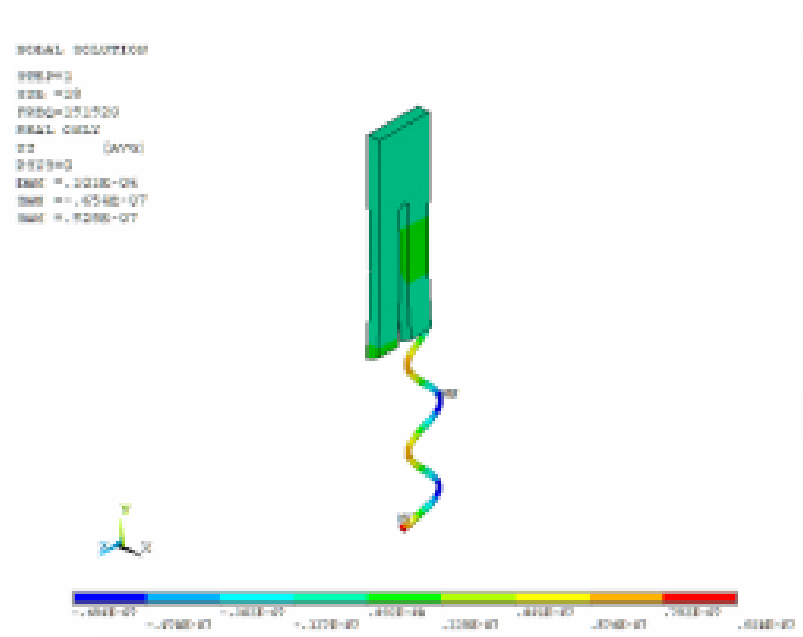

a

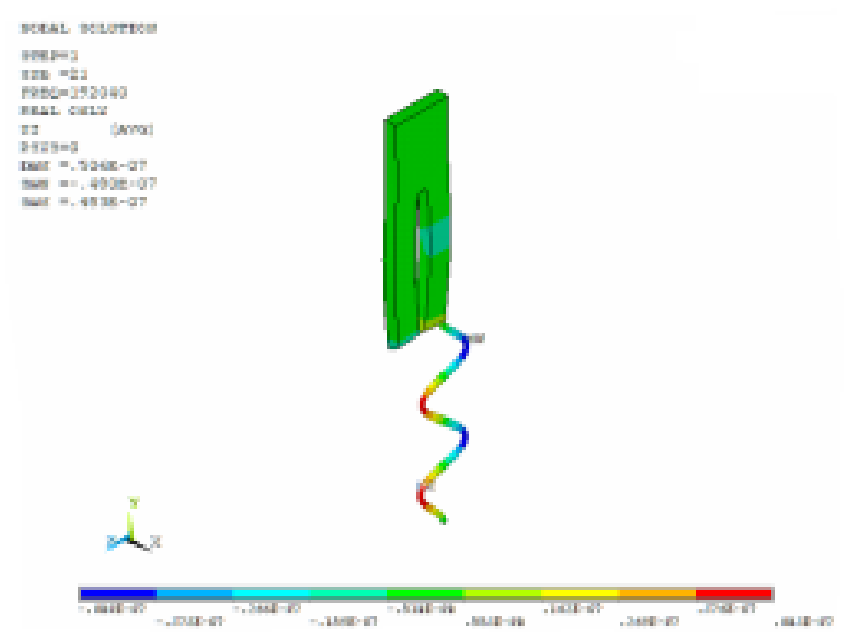

$\mathrm{b}$

Fig. 9 Continued on next page 


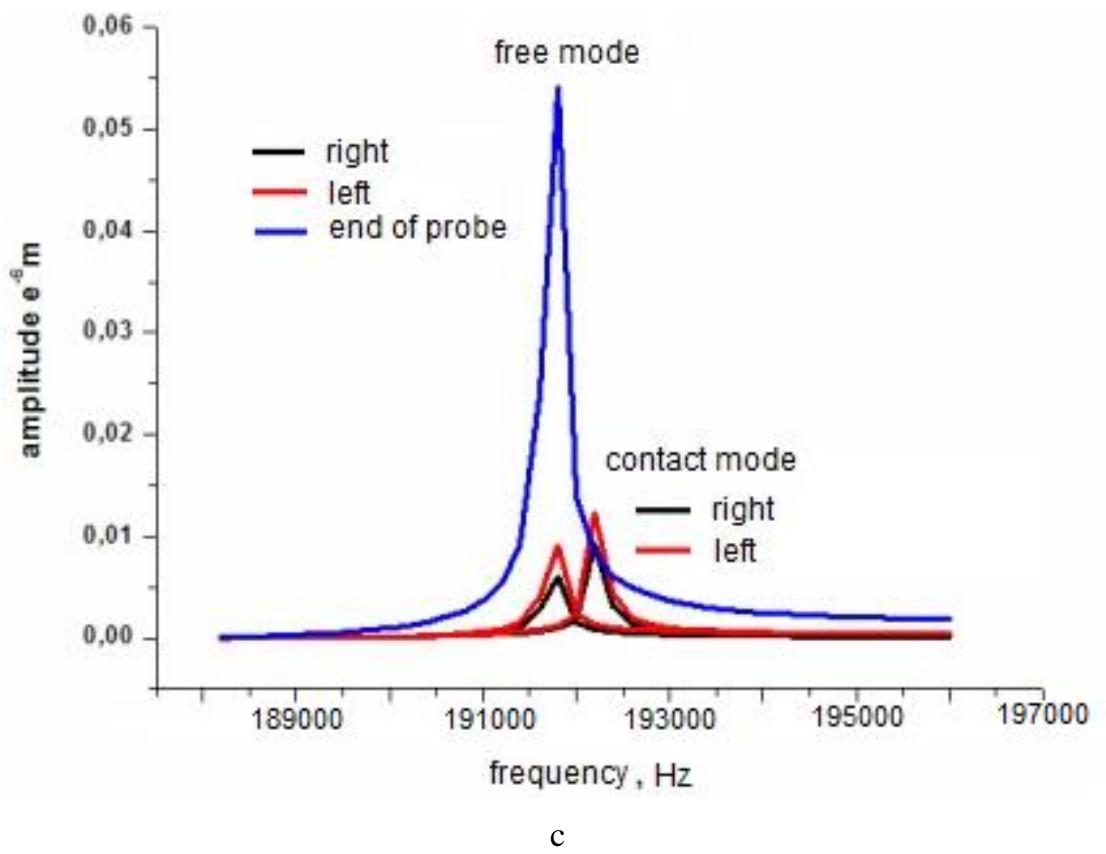

Fig. 9 Tuning fork oscillation resonance frequency $190 \mathrm{kHz}$, tip length $5 \mathrm{~mm}$ a) free contact b) contact with surface c) amplitude-frequency characteristic

The simulation results have shown not linear dependence between the tip length and quartz tuning fork oscillation amplitude, Q-factor and frequency difference.

Simulations results demonstrate that the optimal length have to be selected to get optimal conditions with high Q-factor and optimal length to get maximum frquency shift.

We investigated the possibility to use long probes to get image in liquids. Our experiments demonstrate that the etched tip quality is good enough to perform meas-urements in liquids with a resolution less than $50 \mathrm{~nm}$. The possibility to apply our system to biological samples of Chinese hamster ovary $(\mathrm{CHO})$ cells imaging were investigated. The high quality of images was demonstrated (Fig. 10).

These results suggest that the etched tungsten tips would be a promising tool in for biological applications of shear force and atomic force microscopy with nanopositioning systems [9] and can provide valuable information for understanding some biological problems.

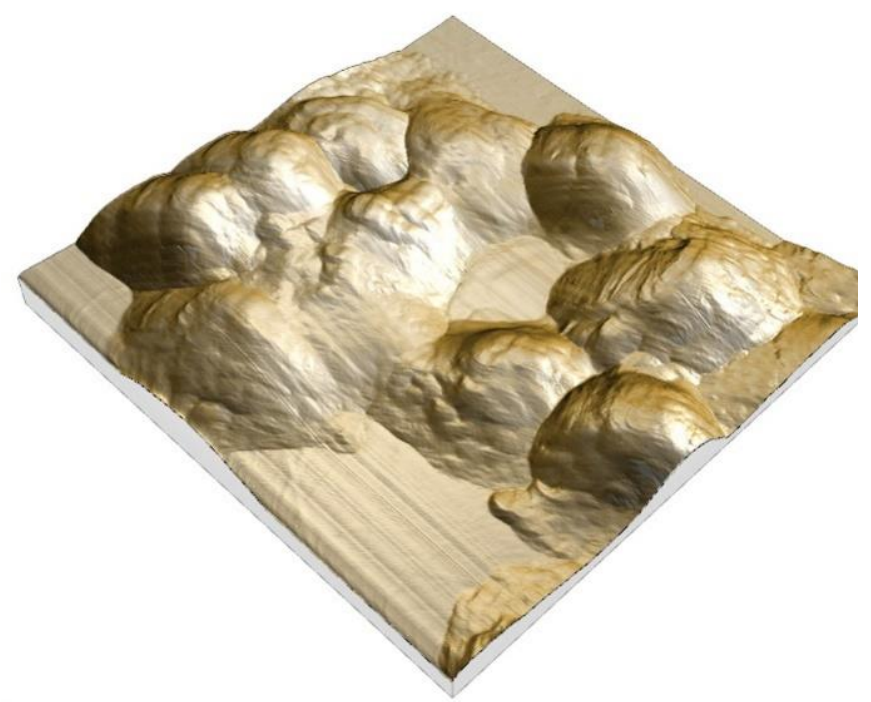

\section{Conclusions}

1. The high sensitivity of the sensor is demonstrated both by shear force and by imaging of soft biological samples in liquid.

2. The experimental results suggest that tuning fork sensor with long long tip would be a promising set-up for the measurements and imaging in liquid.

3. The optimal length of the tip has to be selected for experimental conditions to get a maximum sensitivity. The most sensitive tuning fork at $32 \mathrm{kHz}$ is $2 \mathrm{~mm}$ long oscillation resonance frequency with the free tip in air and with the tip in contact with surface are $2.83 \mathrm{kHz}$. The most sensitive tuning fork at $190 \mathrm{kHz}$ is $0.4 \mathrm{~mm}$ long - oscillation resonance frequency with the free tip in air and with the tip in contact with surface are $2.6 \mathrm{kHz}$.

4. Positive results of testing measurement with the made sensors for shear force microscopy shows, that the developed sensors can be used for shear force microscopy with the resolution less than $50 \mathrm{~nm}$.

\section{References}

1. Karrai, K.; Grober, R. D. 1995. Piezoelectric tip-sample distance control for near field optical microscopes, Appl. Phys. Lett., 66: 1842.

https://doi.org/10.1063/1.113340.

2. Lei, F.; Manfaat, M. 2007. Non-optical bimorph-based force sensor for scanning near-field optical microscopy of biological materials: characteristics, design and applications, Surf. Interface Anal. 39: 674-683. http://doi. 10.1002/sia.2576.

3. Lucier, A. S. 2004. Preparation and characterization of tungsten tips suitable for molecular electronics studies, Center for the Physics of Materials, Department of Physics, McGill University, Montreal, Quebec, Canada: 532.

Fig. $10 \mathrm{CHO}$ cell image: scan area $60 \times 60 \mathrm{~nm}$, height $4 \mu \mathrm{m}$ 
4. Gomer, R. 1961. Field emission and field ionization. Number 9 in Harvard monographs in applied science, Harvard University press: 217-225.

5. Radmacher, M.; Fritz, M.; Hansma, P.K. 1995. Imaging soft samples with the atomic force microscope: gelatin in water and propanol, Biophys. J. 69: 264-270. https://10.1016/S0006-3495(95)79897-6.

6. Takács, G.; Rohal'-Ilkiv, B. 2012. Model predictive vibration control, 1st ed.; Springer: London, UK, p. 87. https://doi.org/10.1007/978-1-4471-2333-0.

7. Bechmann, R. 2008. Elastic and piezoelectric constants of alpha-quartz, Phys. Rev. 110: 1060-1061. https://doi.org/10.1103/PhysRev.110.1060.

8. Ogi, H.; Nakamura, N.; Sato, K.; Hirao, M.; Uda, S. 2006. Elastic, anelastic, and piezoelectric coefficients of alpha-quartz determined by resonance ultrasound spectroscopy, J. Appl. Phys. 100: 3511-3517. https://doi.org/10.1063/1.2335684.

9. Lendraitis, V.; Snitka, V.; Mizariene, V. 2008. Modelling and investigation of the silicon membrane actuator for nanopositioning, Mechanika 2(70): 52-55.
V. Lendraitis

\section{RESEARCH OF QUARTZ TUNING FORK TIP \\ LENGTH INFLUENCE ON THE SHEAR FORCE IMAGING IN LIQUIDS}

S u m m a r y

In this article the use of sensors for shear force microscopy (SFM) is analysed. The sensor consists of a quartz tuning fork with an agglutined long tungsten probe tip for operation in liquid environments.

The quartz tuning fork and a tip oscillation amplitude-frequency dependence in air and a tip in contact with surface were investigated using Finite Element Modeling and experimentally.

The results of this experimental research are valuable in the production of SFM sensors can be produced and used in nanotribology, nanolithography, nanometric acoustic spectroscopy and biological object research.

Keywords: quartz tuning fork, finite element model, liquids, shear force microscope.

Received September 28, 2017 Accepted February 15, 2018 